\title{
Digital Visualisation and Design Development in Historic Building Projects
}

\author{
Vajira Premadasa \\ Major Projects Team \\ Historic Scotland \\ Edinburgh EH9 1SH \\ UK \\ vajira.premadasa@scotland.gsi.gov.uk
}

\author{
Michael Pearce \\ Department of History \\ University of Dundee \\ Dundee DD1 4HN \\ UK \\ mpearce@dundee.ac.uk
}

\begin{abstract}
The design stages of projects involving high-profile historic buildings often present a unique set of challenges to architects and allied professionals. It is crucial to carefully balance rigorous cultural significance and academic criteria with modern requirements and standards, resulting in a need to investigate and refine a wide range of design options before work can commence on site. Although digital visualisation techniques are commonly used in built heritage as a means of interpreting historic buildings and sites, they can also be a powerful and flexible design development and management tool in conservation and refurbishment projects. Indeed their utility extends beyond straightforward visual design investigation to include such areas as product specification, cost control, quality assurance and change management at later project stages. This paper presents the Stirling Castle Palace Project as a case study in the application of these techniques, and also investigates ways in which convergent trends in digital data acquisition, information management and immersive systems can contribute to the formulation of a comprehensive digital workflow for historic building projects.
\end{abstract}

Building conservation. Digital visualisation. Historic interiors.

\section{INTRODUCTION}

The use of digital visualisations in built heritage applications has progressed from what Reilly (1992) described as a 'flirtation with an exotic technology' to a situation where it is an increasingly indispensable tool for the interpretation of the past. Some of the early visualisations of historic buildings carried out in the 1980s were indeed conceived as test beds for new technologies. They were often implemented using industrial software which required input in the form of code due to the absence of graphical user interfaces, but which nevertheless managed to produce some impressive results. Following this adoption phase, an early example of the use digital visualisations and immersive technologies for the provision of enhanced visitor interpretation was documented by Boland \& Johnson (1996) in their description of a virtual tour of Dudley Castle.

Concurrently with the use of digital visualisations for public interpretation there has been an increasing trend towards their utilisation in the academic investigation of historic buildings and sites (Gifford \& Acuto 2002), along with their application to the design development stages of historic building projects. In the latter context, where the emphasis is primarily on critical visual analysis rather than the communication of established knowledge, a fundamentally different set of performance criteria must be satisfied. The overall visualisation methodology has to be flexible enough to enable design proposals to be quickly tested, but also adequately robust to support the academic discourse underlying the design development process.

Historic Scotland, an Executive Agency of the Scottish Government tasked with the care and management of Scotland's built heritage, recently completed a prestigious project to refurbish and present the historic royal apartments at Stirling Palace as they may have originally appeared in the 16 th century. The refurbishments were based on the findings of a thorough academic research programme which also included the development of comprehensive design proposals for the interiors of the apartments. The early stages of the project focused on testing and progressively refining the proposals with the aid of detailed digital visualisations, which were subsequently used as contractual documents to specify the standard of workmanship required for the implementation stage of the project. As will be described in this paper, the 
digital visualisations played a key role in the eventual success of the project.

\section{THE HISTORY OF STIRLING PALACE}

The palace at Stirling Castle was built around 1540 for James $V$ of Scotland and his French queen Mary of Guise. The rectangular block incorporates part of the forework of the castle and compliments the King's Old Building and the Great Hall, three additions to the castle built around 1500 by James IV. The plan appears novel in conception for Scotland at the period, serving to accommodate two nearly symmetrical suites of state apartments for James and Mary of equal proportions around a central square. In other Scottish palaces the suites were stacked over each other, and this Renaissance arrangement is of particular significance as a unique survival in Britain (Figure 1).

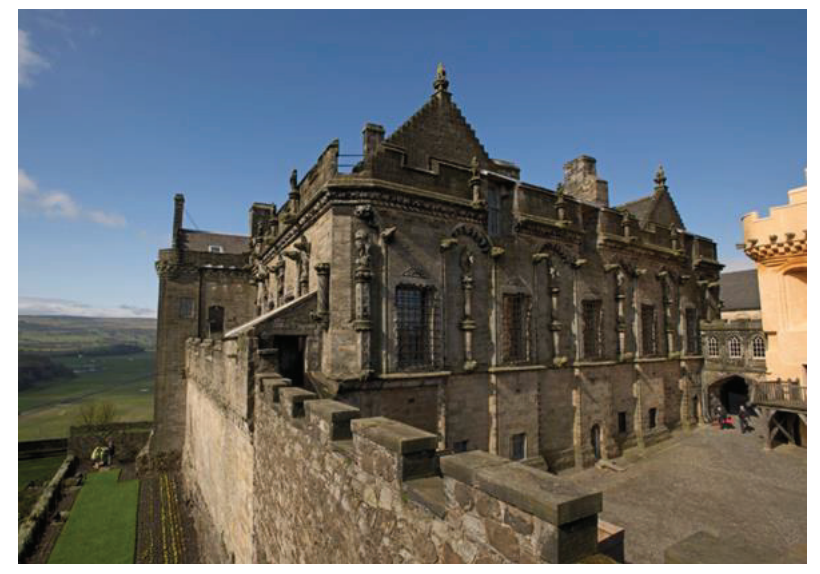

Figure 1: Stirling Palace (Michael Brooks)

Each royal suite contains a bedchamber, an inner hall or audience chamber, and an outer hall. The west quarter contained a gallery and lobby linking both suites, but only survived in a re-worked and less intact state. However the masonry of the other three façades and room divisions of the state rooms within remains as built around 1540. The exterior has a series of statues alternating in arcaded niches with the windows. The character, detail and design source of this sculpture is also of Renaissance inspiration; in spite of groundbreaking research carried out by the University of Glasgow (Rush 2011), the meaning of the sculptural scheme remains somewhat conjectural, though it was obviously intended to celebrate the prestige of the royal Stewart dynasty of Scotland.

The interiors of the state rooms suffered major damage in the 17th century. During the Civil War the Castle was besieged and later some of the rooms in the Palace were made into granaries, and then barracks. The celebrated carved oak ceiling of the king's audience chamber was dismantled in 1777 , although many of the portrait roundels now known as the Stirling Heads, which date from the 1540 s, survived. By the end of the 18th century the state rooms were filled with ranks of bunk beds for soldiers, which were then removed in the 19th century and the rooms made into mess quarters with a regimental museum above. Despite all these changes and losses to the interiors it was discovered in 2005 that a number of the heavy oak doors dated from around 1540 and were still in-situ.

Although used as an army depot the castle had always welcomed visitors keen to appreciate its historical associations, and a number of 18th century visitors recorded the fine ceilings of the palace. In the 1930s the Chapel Royal was cleared of later accretions and its interior partially restored, with the whole Castle becoming a monument in state care in 1964. The Great Hall of James IV was returned to its original appearance in 1998, and in 2003 work began to investigate the structural integrity and archaeology of the Palace of James $\mathrm{V}$. The fittings of the interiors installed since 1964 were stripped along with damaging cementitious plaster to reveal the original fabric, all of which was then carefully recorded by architectural and archaeological survey as a preparatory stage to the commencement of work on site to refurbish the interiors of the royal apartments.

\section{THE PALACE PROJECT}

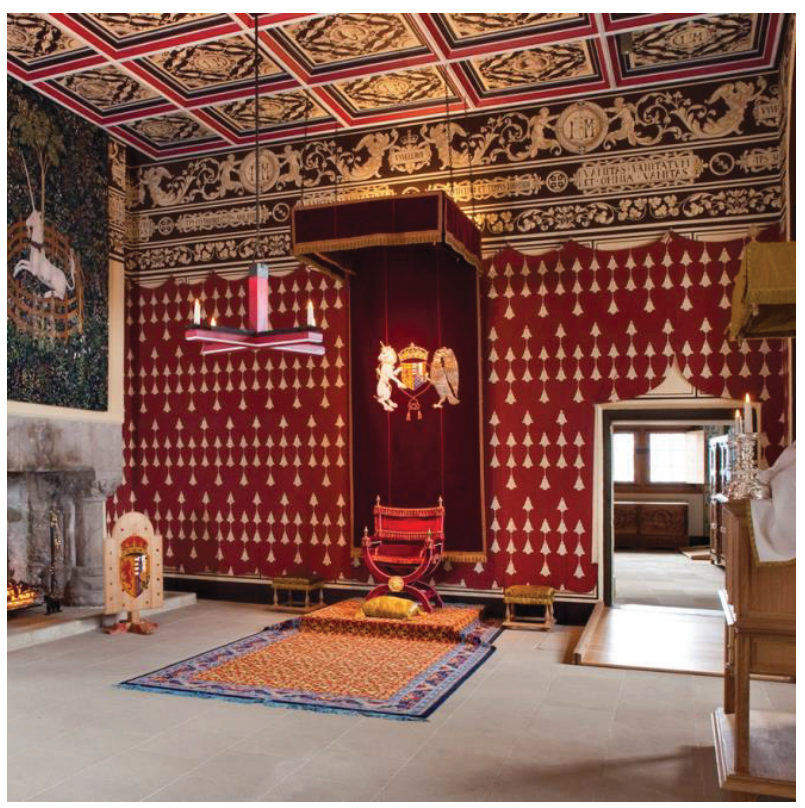

Figure 2: The Queen's Inner Hall after completion (Michael Brooks)

The Stirling Castle Project was conceived in the early 1990 s as a multi-phase initiative to enhance the visitor experience at what was then fast becoming a major tourist attraction. Whereas the 
initial phases of the project focused on the provision of improved castle-wide visitor facilities followed by the refurbishment of the Great Hall, Phase IV involved the redevelopment of the palace itself with the objective of reflecting its status as one of the finest surviving Renaissance buildings anywhere in Britain. Preceded by an extended design development phase, on-site work commenced in 2008 and the completed project was opened to the public by H.M. the Queen in July 2011 (Figure 2).

The intention from the outset was to refurbish and present the interiors of the palace as they may have appeared when they were newly finished in the 1540 s, both on the basis of surviving evidence within the palace itself and also the royal inventories of furniture dating from the 1540s and 1580s. Neither of these sources is exhaustive, given that not many original features survive, and that the inventories are fragmentary and do not provide complete information on the various items described in them (Harrison 2011). Consequently the Stirling Palace Academic Research Committee (SPARC), which was formed in 2003 , sifted through the surviving evidence to formulate a rigorously compiled specification of furnishings, fittings and decorative elements. Whatever information was missing was supplemented by contemporary documentary, pictorial and physical evidence that closely corresponded with their descriptions in the inventories. In addition, much as was done at the time when the palace was originally built, 16th century pattern books were used to extract decorative references with a conscious attempt being made to reflect the classicising, distinctly French influence that the palace embodies. The results of this academic research exercise were subsequently made available to the public through the Scran online resource managed by the Royal Commission on the Ancient and Historic Monuments of Scotland (RCAHMS).

Each of the items in the specification was then classified into a series of contract work packages (joinery, metalwork, stained glass, etc.). These work packages were subsequently tendered in accordance with UK Government procurement guidelines to enable the appointment a team of crafts contractors who were specialists in their particular fields. In coordination with Historic Scotland's own specialist staff, this team of contractors developed detailed designs for the individual specification items, and after design approval, manufactured and installed the items on site. A significant element of coordination was required to ensure that the project was completed to programme, principally because many of the specification items required the involvement of multiple contractors.
All this could prompt the question as to whether the Stirling Palace Project involved the making of a conjectural reconstruction on the basis of incomplete evidence, a practice which the Venice Charter (the key document relating to the conservation of cultural heritage) highlights as an approach to be avoided. What should be noted, however, is that the palace interiors are not reconstructions but rather entirely new creations, designed and executed within the constraints of a rigorous academic framework. Nor was any attempt made to 'antique' the interiors or the fittings to give them a spurious appearance of authenticity; the refurbished interiors are presented as frankly modern interpretations of what could plausibly have existed in the 1540s, and this is clearly explained to visitors. In addition, all the work was carefully carried out so as not to damage the original fabric in any way and also to be completely reversible should it be decided at some future date to remove the refurbished interiors.

\section{VISUALISING THE ROYAL APARTMENTS}

Given the high level of complexity involved in formulating a historically informed internal decorative scheme for the royal apartments, it was necessary to undertake an extended design development process to examine a range of options and to iteratively refine them as new research information was made available by SPARC. Even though early scheme designs were illustrated by means of hand-drawn visuals, it was clear that in spite of their undeniable appeal this particular method was too static and rigid to be used as a design development tool in a project of this nature. Consequently the decision was made to use digital visualisations for this purpose on the basis that not only was this approach more flexible, but also because it was anticipated that the visualisations would aid in marketing the project and could eventually be used to provide enhanced visitor interpretation at the castle. As a result, Fleming Woelfell Imaging Ltd was employed by Historic Scotland in 2005 to produce the visualisations of the royal apartments in liaison with SPARC.

At this early stage of the design process the visualisations were commissioned as still images, not only because this was a quick method to test alternative design options, but also because the images could be easily disseminated to the various project stakeholders for discussion and eventual approval. A further reason was that it allowed SPARC to focus on specific areas of the proposed design in a way that would be difficult or impossible with moving images. It should be noted that the visualisations were not meant to convey an absolute knowledge of what may have existed in 
the 1540 s, or indeed to give a definitive impression of what the eventual end product of the project might be. Instead, they were starting points for the various specialist crafts contractors employed on the project to develop their own designs in keeping with the aesthetic principles of the Scottish Renaissance.

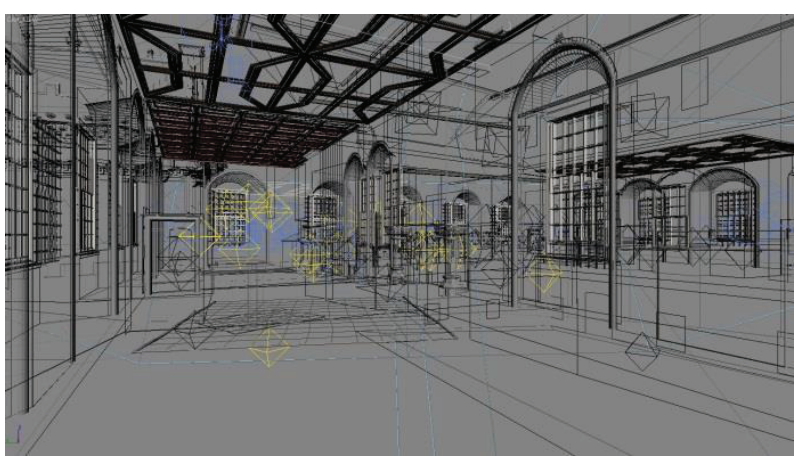

Figure 3: Digital model of the royal apartments (Fleming Woelfell)

The visualisation process involved making a detailed digital model using 3DS Max software based on a set of comprehensive orthographic survey drawings, which consisted of both traditional hand surveyed material and also electronic distance meter (EDM) survey data (Figure 3 ). It is notable that this survey data was not rationalised during the modelling process; the highly irregular existing fabric was modelled as faithfully as possible, with no simplification to make the model more manageable. In any case as the model was intended to be used as a static design development tool and not as the basis for an interactive experience or animation where a low polygon count and consequent high rendering speeds would be paramount, it was possible to maintain a close correspondence with the original survey data even at the cost of a more geometrically complex model.

The model was rendered with the standard 3DS Max rendering engine, using the careful arrangement of lighting to soften and add depth to the visualisations. The resulting visualisations were then analysed and refined at regular design development meetings; any changes arising from these discussions were then made to the model in order to iteratively formulate a historically plausible interior decoration scheme for the royal apartments (Figures 4 \& 5). In addition to modelling the building itself, all the individual items of furniture were modelled on the basis of historical source information provided SPARC. This enabled each item to be assessed in isolation and also to be seen as part of a complete decorative scheme. The 37 specially carved replica Stirling Heads installed on the ceiling of the King's Inner Hall were one of the most significant highlights of the recreated royal apartments. Potential paint schemes for each of the heads were carefully tested out, in addition to which the entire ceiling was visualised to give an impression of how the heads would appear as a group (Figure 6).

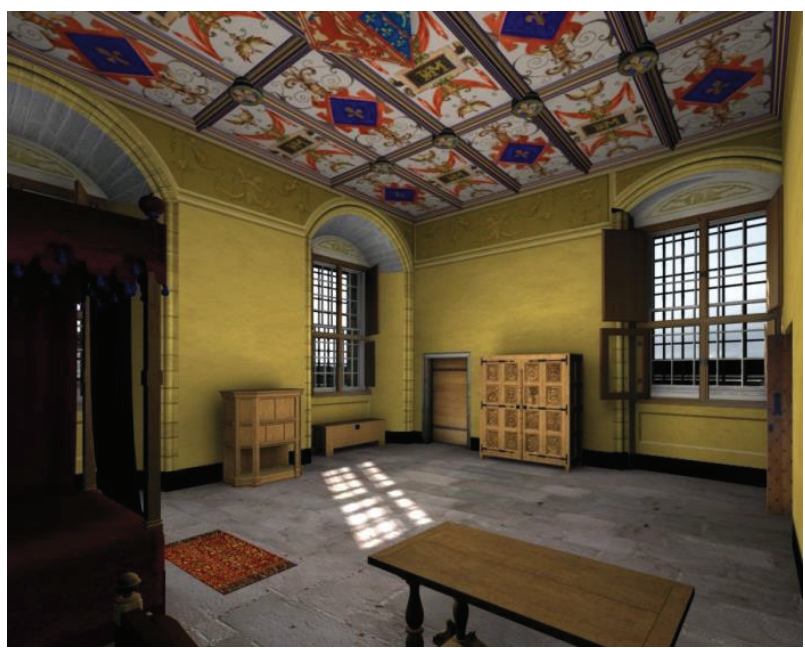

Figure 4: Design development visualisation for the Queen's Bedchamber (Fleming Woelfell)

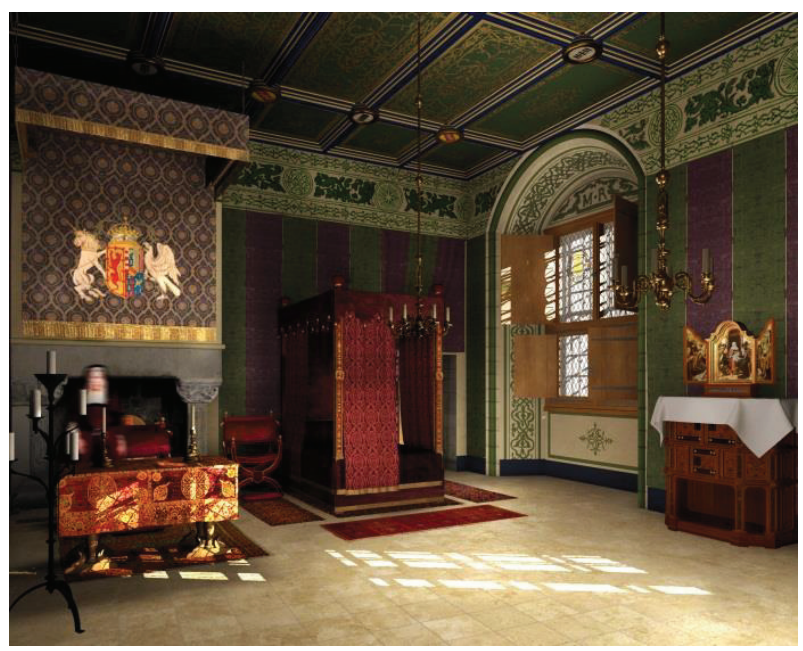

Figure 5: Final design scheme visualisation for the Queen's Bedchamber (Fleming Woelfell)

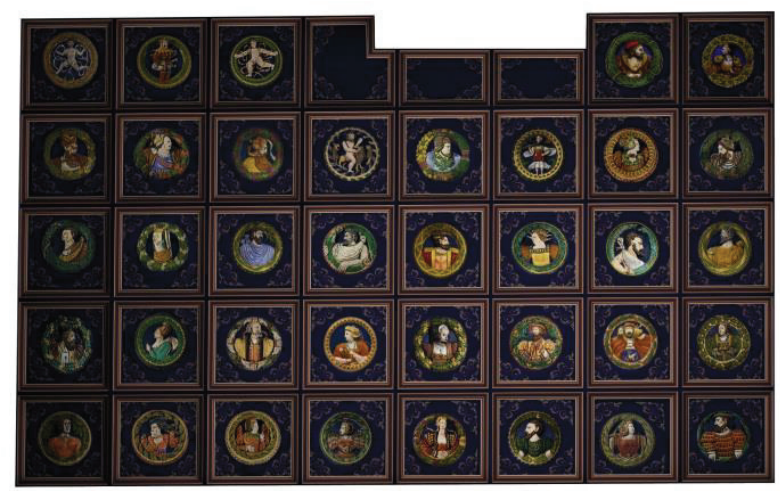

Figure 6: Visualisation of the Stirling Heads on the ceiling of the King's Inner Hall (Fleming Woelfell) 
Another crucial area where the visualisations were found to be indispensable was in the development of the lighting design scheme for the royal apartments. The apartments would originally have been lit by beeswax candles, giving much lower overall lighting levels than those we are accustomed to today. Whilst there is little doubt that recreating these levels would have imparted an added level of authenticity to the recreations, it was clearly impractical to do so, mainly due to concerns with visitor safety. Consequently extended design studies were carried out by Foto-Ma Lighting Architects in collaboration with Fleming Woelfell Imaging Ltd to establish suitable lighting levels that would strike a balance between providing adequate lighting for visitors whilst at the same time giving a close approximation of what may have originally existed. These design studies were then used to inform the specification of appropriate luminaires that would provide the required lighting levels (Figure 7).

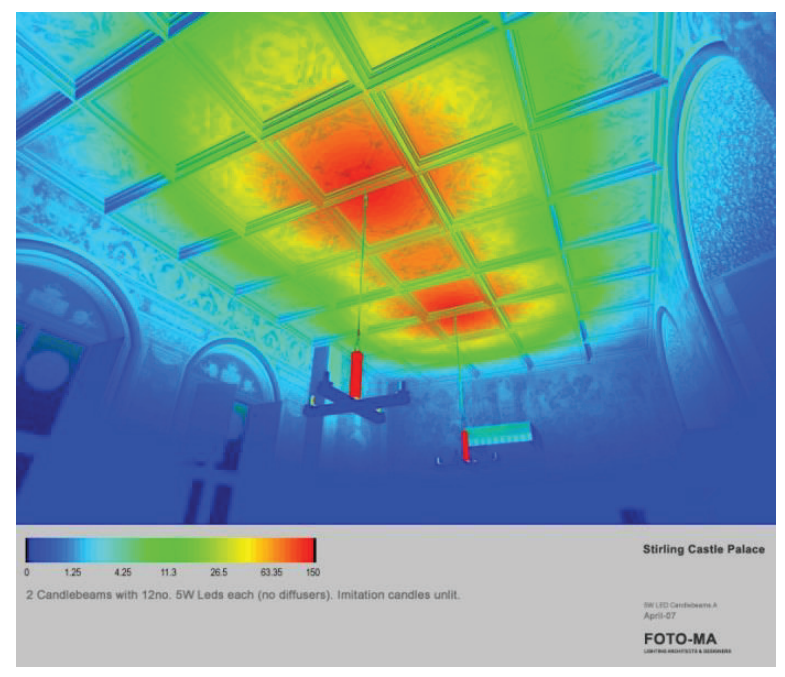

Figure 7: Testing lighting levels in the Queen's Inner Hall (Fleming Woelfell \& Foto-Ma)

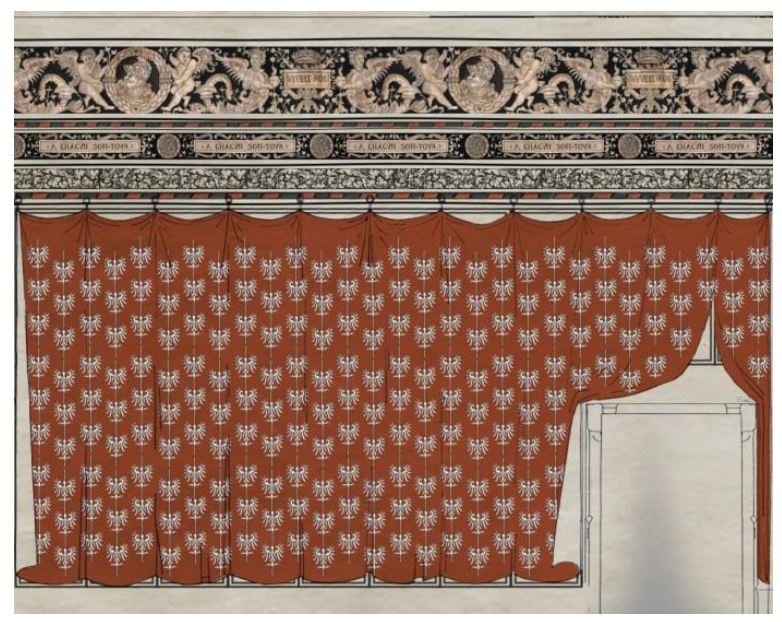

Figure 8: Texture map of the applied wall decoration in the Queen's Inner Hall (Fleming Woelfell)
The visualisation workflow included a significant element of texture, transparency and displacement mapping, principally in the case of the stained glass panels, fabric wall hangings and applied wall decoration (Figure 8). The maps were created by referencing appropriate original sources which were then copied and painstakingly painted, both by hand and using image manipulation software. It is also noteworthy that the fully mapped visualisations were used for material take off during the procurement phase of the project, particularly in the quantification and costing of the amount of gold leaf that would be required for the ceiling of the Queen's Bedchamber (Figure 9).

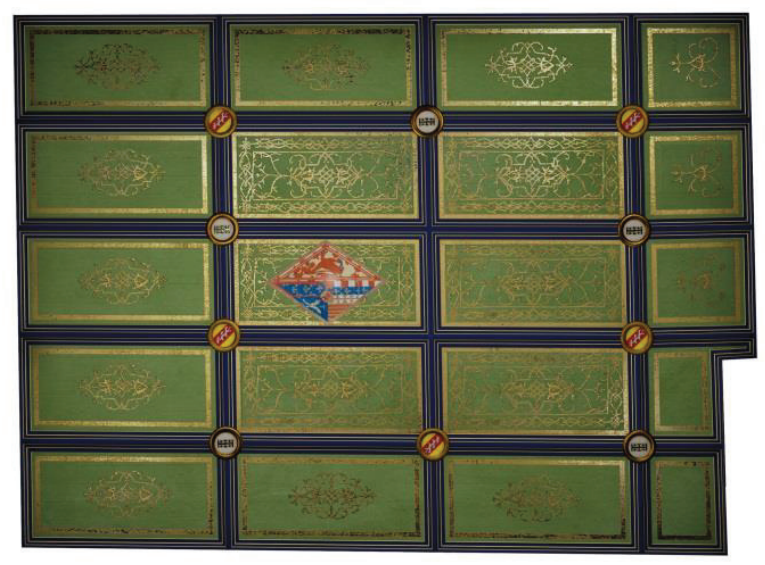

Figure 9: Ceiling of the Queen's Bedchamber (Fleming Woelfell)

Thus the visualisations contributed to establishing cost certainty and budgetary control on certain elements of the project, in addition to playing a crucial role as contract documentation during the initial tendering process for the appointment of the crafts contractors. A final point to be made is that the visualisations continued to be used for the duration of the project as a quality control and change management tool against which the performance and costs of the crafts contractors were evaluated.

\section{OBSERVATIONS AND APPRAISAL}

There is little doubt that the digital visualisations of the palace interiors contributed in a major way to enabling a complex historic building refurbishment project to be successfully completed to an extremely high standard. Nevertheless it should be remembered that the visualisations were produced between 2006 and 2010 (with much of the work being carried out towards the front end of the programme) and were therefore constrained in certain areas by the limitations of the technology commonly available at the time. It is perhaps pertinent to highlight a number of these areas and to discuss ways in which their shortcomings could 
potentially be dealt with in future projects of this nature.

\subsection{Data acquisition}

It has been mentioned that the digital model of Stirling Palace was based on a pre-existing set of comprehensive orthographic survey drawings, which in turn were based on source data obtained over several years either by hand measurement or latterly by EDM survey. As a result this essentially two-dimensional dataset had to be converted into a three-dimensional one, involving a lengthy and inefficient workflow with increased scope for the introduction of metric inaccuracies. Therefore although the interiors model was a faithful reflection of the original survey data, the fact remains that any model constructed in this way will always be an approximation of the actual building purely for the reason that it is impossible to accurately represent a complex three-dimensional object using twodimensional media without the loss of metric information.

It is therefore crucial to obtain and record source data in an inherently three-dimensional format and to eliminate intermediate data translations if metric accuracy is to be preserved. In current historic building practice this would usually involve the implementation of a laser scan survey, whether terrestrial, aerial, or a combination of the two. In fact the use of laser scanning in large built heritage applications is now fairly widespread, not only for its inherent three-dimensionality and high level of metric accuracy, but also because the scan data allows for a range of subsequent operations including condition monitoring and spatial analysis (English Heritage 2011). However it should be noted that laser scanning equipment and software remains expensive (although this is changing), and there is also a significant post-processing element involved in converting laser scan point clouds into a more convenient and manageable format.

As an aside, while the Stirling Palace Project was nearing completion in 2010 Historic Scotland carried out a laser scan survey of the Palace in partnership with the Digital Design Studio of the Glasgow School of Art. The laser scan data was then used as the basis of a conjectural reconstruction of what the exterior of the Palace may have looked like in the 16th century, and was also used to create an animation of the Palace. However due to the fact that the laser scan survey had not yet been carried out at the inception of the Palace Project, the model of the royal apartments was not able to benefit from this point cloud data. Having said that, it would nevertheless be a very worthwhile future exercise to examine the possible integration of what are currently two completely disparate datasets to generate a complete model of both the interior and the exterior of the palace.

\subsection{Building Information Modelling}

The model of the palace interiors was created using straightforward constructive solid geometry for the fundamental reason that it was conceived as a design development tool with limited relevance to the subsequent construction and long-term management phases of the project. Thus there was no requirement to create a parametric model incorporating intelligent object behaviour which could then be developed into a mature Building Information Modelling (BIM) workflow. In spite of the fact that the UK Government has committed to the use of collaborative 3D BIM on all its projects by 2016, a universally accepted definition of BIM remains elusive. The one offered by Penttilä (2006) perhaps captures the essential underlying concept: he defines BIM as 'a methodology to manage the essential building design and project data in digital format throughout the building's life-cycle'.

Although a detailed discussion of BIM is beyond the scope of this paper, it should be noted that in spite of some recent research in this area (Fai, Graham et al. 2011, Murphy, McGovern et al. 2011) the use of BIM on historic building projects, particularly those of high cultural significance, is still very much in its infancy. Historic Scotland is currently in the early stages of developing a BIM deployment plan for its major construction capital projects, anticipating that this would contribute to significantly improved design visualisation, construction information coordination and eventual long-term management of the sites in its care. A particular area of interest is the use of BIM for clash detection and coordination between various building elements. As the majority of historic building projects involve the careful introduction of modern structural and servicing elements with minimal alteration to the existing building fabric, the detection and rectification of potential clashes during the design stage would undoubtedly play a part in establishing a more efficient and error-free workflow during the implementation stage.

Historic Scotland is also investigating the development of a protocol to facilitate the seamless transfer of laser scan point cloud data into BIM software in order to preserve the integrity of threedimensional information acquired on site. The importance of metric accuracy has already been stressed, but it becomes all the more crucial to ensure that original source data is retained uncorrupted when it is used as the basis for a comprehensive multi-purpose building model. Furthermore, as explained by El-Hakim, Beraldin et al. (2005), modelling a large heritage site often 
involves the combination of datasets from a variety of sources, not all of which are likely to be immediately compatible with each other. This was the case at Stirling Palace, where in addition to the modern survey data there was a wide variety of information contained in historic survey drawings. Partly due to the reason that most of this information was not readily transferable to $3 \mathrm{D}$ format and also because its metric accuracy could not be depended on, it was generally used only for reference purposes. Since then however, as part of its planned adoption of BIM methodologies on future capital projects, Historic Scotland plans to investigate the development of formal systems to integrate historic building information with digital building models.

\subsection{Augmented reality}

Although in the case of the Stirling Palace Project the decision was made to present the visualisations of the interiors in the form of still images, it is evident that this particular medium has many significant drawbacks as a design development tool. The most serious of these is that a still image cannot accurately represent the threedimensionality of the space being portrayed, and consequently such an image cannot be anything but an abstraction of reality. On the other hand as Augmented Reality (AR) essentially supplements and enhances reality by mapping computergenerated information onto the user's view of the real world (Champion 2011), an AR-based design development tool would enable the threedimensional superimposition of the design proposals onto the existing building fabric. Therefore such a tool would allow the design team to dynamically assess the proposals in their realworld setting, and by doing so to balance the various project constraints by direct reference to the actual three-dimensional characteristics of the spaces in question.

Another area where AR-based tools could potentially prove to be extremely useful in historic building projects is during the site operations stage, particularly as many coordination errors and delays stem directly from the fact that construction information is still generally represented and communicated by means of paper drawings (Cote 2012). The impossibility of trying to represent threedimensional information using two-dimensional media has already been explained, but what is particularly relevant is that historic buildings in general tend to be far more geometrically irregular than modern ones, which has the effect of exacerbating the problem and making site settingout in particular a very challenging task indeed. This is all the more crucial as it is often the case in historic building projects that modern elements have to be inserted into existing building fabric with minimal impact. On the Stirling Palace Project, for instance, it was necessary to set out and insert a new steel floor structure into the existing timber floor without causing any damage to the historic joists, which in the event turned out to be an extremely complex and protracted operation. The use of an AR-based site setting-out tool with the capacity to map and visually identify key points directly on the existing building fabric would have enabled this operation to be carried out in a much more reliable and efficient manner than attempting to do so manually. Indeed it is not difficult to imagine that the combined use of such a tool with BIM clash detection routines at the design stage would have contributed greatly to the elimination of coordination errors.

\section{CONCLUSION}

The Stirling Palace Project was Historic Scotland's first attempt at the intensive application of digital visualisation tools in the design development stage of a major capital project. It is fair to say that, although the visualisations played a key role in contributing to the eventual success of the overall project, the exact methodology that was adopted left several unanswered questions. The key ones were the following:

- How can data be acquired on site in an inherently three-dimensional format and subsequently transferred in a loss-free manner to create the building model?

- How can historic source data be incorporated into this process?

- How can the building model be used for construction and long-term building management, in addition to its role as a design visualisation tool?

- How can the building model be visualised, interrogated and refined in a dynamic and interactive manner while maintaining a close correspondence with the existing building fabric?

These questions are common to many major historic building projects, particularly where the building in question has a particularly high cultural significance. As described above, the development of a standard project workflow incorporating recent developments and convergent trends in laser scanning, BIM and augmented reality may in fact provide a very effective means to address these issues. Future advances in such areas as wearable computing and 3D printing may well revolutionise the field even further.

It is important, however, to sound a note of caution. Throughout the relatively short history of digital heritage there has perhaps been a tendency to 
view technology as a panacea for all ills. For instance one of the first concerns raised in this respect was that many of the early digital reconstructions of historic buildings were more concerned with photorealistic image-making than with the intellectual integrity of the underlying content (Miller \& Richards 1995), as a consequence of which they often took on an unjustified appearance of definitive knowledge. Therefore it is as well not to lose sight of the fact that the ultimate role of digital technology in built heritage applications is to support the documentation, interpretation and management, not of abstract concepts, but of what are profoundly human cultural expressions.

\section{REFERENCES}

Boland, P., and Johnson, C. (1996) Archaeology as Computer Visualisation: 'virtual tours' of Dudley Castle c.1500. In Higgins, T., Main, P., \& Lang, J. (eds), British Museum Occasional Paper No. 114: Imaging the Past. British Museum Press, London.

Champion, E. (2011) Playing with the Past. Springer, London.

Cote, S. (2012) Augmented Reality for Building Construction and Maintenance: Augmenting with 2D Drawings.

http://communities. bentley.com/other/old site mem ber blogs/bentley employees/b/stephanecotes blo g/archive/2012/09/11/augmented-reality-forbuilding-construction-and-maintenanceaugmenting-with-2d-drawings.aspx (retrieved 31 March 2011).

El-Hakim, S., Beraldin, J.-A., Gonzo, L., Whiting, E., Jemtrud, M., and Valzano, V. (2005) A Hierarchical 3D Reconstruction Approach for Documenting Complex Heritage Sites. CIPA 2005 International Symposium, Turin, 26 September 01 October 2005.

http://cipa.icomos.org/fileadmin/template/doc/TURI N/790.pdf

(retrieved 11 January 2013).

English Heritage (2011) 3D Laser Scanning for Heritage: Advice and Guidance to Users on Laser Scanning in Archaeology and Architecture.

http://www.english-heritage.org.uk/publications/3dlaser-scanning-heritage2/

(retrieved 21 March 2013).

Fai, S., Graham, K., Duckworth, T., Wood, N., and Attar, R. (2011) Building Information Modelling and Heritage Documentation.

http://www.autodeskresearch.com/publications/herit agedoc

(retrieved 11 January 2013).
Gifford, C., and Acuto, F. (2002) Space, Place and Inka Domination in Northwest Argentina. In Mathieu, J.R., (ed) Experimental Archaeology: Replicating Past Objects, Behaviours and Processes. Archaeopress. Oxford.

Harrison, J. G. (2011) Rebirth of a Palace, Historic Scotland.

ICOMOS (1964) Venice Charter for the Conservation of Monuments and Sites.

http://www.international.icomos.org/charters/venice e.pdf

(retrieved 25 March 2013).

Miller, P., and Richards, J. (1995) The Good, the Bad and the Downright Misleading: Archaeological Adoption of Computer Visualisation. In Huggett, J. \& Ryan, N., (eds) Computer Applications and Quantitative Methods in Archaeology 1994. Tempus Reparatum, Oxford.

Murphy, M., McGovern, E., and Pavia, S. (2011) Historic Building Information Modelling - Adding Intelligence to Laser and Image Based Surveys. ISPRS Trento 2011 Workshop, Trento, 2 - 4 March 2011.

http://www.int-arch-photogramm-remote-sensspatial-inf-sci.net/XXXVIII-5-

W16/1/2011/isprsarchives-XXXVIII-5-W16-1-

2011.pdf

(retrieved 11 January 2013).

Penttilä, H. (2006) Describing the Changes in Architectural Information Technology to Understand Design Complexity and Free-Form Architecture. In Howard, R., (ed), Journal of Information Technology in Construction, Vol. 11.

http://www.itcon.org/data/works/att/2006-

29.content.02253.pdf

(retrieved 07 March 2013).

Reilly, P. (1992) Three-dimensional Modelling and Primary Archaeological Data. In Reilly, P., \& Rahtz, S. (eds), Archaeology and the Information Age: A Global Perspective. Routledge, London.

Rush, S. (2011) Heads and Sculpture. Stirling Castle Palace Project - 'Rebirth of the Palace', Stirling, 23 November 2011.

Stirling Castle Palace Archaeological and Historical Research.

http://sparc.scran.ac.uk/home/homePage.html (retrieved 30 March 2013).

UK Government Cabinet Office (2011) Government Construction Strategy.

https://www.gov.uk/government/uploads/system/upl oads/attachment data/file/61152/GovernmentConstruction-Strategy 0.pdf (retrieved 25 March 2013). 